\title{
Studying the Utility of Tracking Systems in Improving Healthcare Workflow
}

\author{
Eiman Elnahrawy and Richard P. Martin \\ \{eiman,rmartin\}@cs.rutgers.edu \\ Department of Computer Science, Rutgers University \\ 110 Frelinghuysen Rd, Piscataway, NJ 08854
}

\begin{abstract}
This paper describes tracking experiments we ran in medical settings in order to study the utility of RTLS systems in modeling hospitals workflows in real time. It reports some lessons we learned regarding the density of the tracking landmark deployment as well as the power and mounting of the tracking tags and their effect on the tracking accuracy. It then discusses how we deduced the clinical activities along the workflow from the RTLS tracking data.
\end{abstract}

\section{INTRODUCTION}

Although the United States spends a full 5\% of its Gross Domestic Product (GDP) more than comparable industrialized countries on health care, it is not a leader in health outcomes. Current health care delivery systems leave much room for improved efficiencies. Several clinical studies have shown that 20\%-50\% of care providers' time is spent on overhead as opposed to direct or indirect care-related tasks. To identify workflow bottlenecks and efficiencies currently requires costly, labor intensive time-and-motion studies.

Recent advancements in wireless technology and the increase in affordability of key hardware components have enabled cost-effective, scalable tracking of mobile objects such as care providers and equipment in indoor spaces. Accurate tracking of all those entities inside large medical centers offers the opportunity to automatically convert spatial-temporal traces of patient, staff and equipment into meaningful clinical events. Further analysis would then map sequences of such events to patientcentered workflows in real time. Such an automated system has several applications, including better realtime communication of status between different units in a hospital, allowing administrators to make better staffing decisions on both a short term and long-term basis, and also make better inventory decisions. All these applications would improve patient throughout and reduce the cost of care delivery.

In this paper we provide initial results and lessons learned using a Real Time Location System (RTLS) in clinical environments for the purposes of automating workflow event detection. We first describe our deployment and results in a cancer clinic. We next describe our experiments emulating real Emergency Department (ED) workflow scenarios. We show that current state- of-art RTLS technologies are quite promising; they can give good detection rates with very low false positives. Our initial finds show that for workflow applications, the RLTS should perform room-level discrimination with a high accuracy, over $99 \%$. However, such accuracy can be obtained over several-minutes timescales, and so the instantaneous accuracy can be lower because a variety of smoothing and filtering algorithms can successfully trade latency for accuracy.

We also found special care must be taken during deployment. In particular, we had to double the density of wireless readers from the cancer clinic to the ED scenario, to approximately one every $400 \mathrm{sq} \mathrm{ft}$. We also found that the RTLS was sensitive to proper signal strength transmission and reception; poor quality antennas, connectors and tag placement had significant impacts on the localization accuracy. As such, we found that software to quickly identify attenuated signals was critical to obtaining good results.

The rest of this paper is organized as follows. Section II gives a brief background of the tracking RTLS system we used in our study. In Section III we describe our deployment in a cancer clinic. Section IV then describes a more extensive experiment we conducted after tuning the RTLS system as well as our approach to deducing clinical events from locations information. Section V describes some of our future research directions. We discuss related work in Section VI. Finally, in Section VII we conclude.

\section{BACKGROUND: GRAIL}

We start with a brief background of the real time tracking system (RTLS) we used in our experiments.

The General purpose, Real-time Adaptable Indoor Localization (GRAIL) RTLS is an open source project developed from the early positioning work done at Rutgers University [5], [6], [3]. GRAIL is designed to be a general purpose platform that uses Received Signal Strength (RSS) modality for tracking and supports a range of different radio technologies, positioning algorithms, user-interfaces and higher-level applications.

We were motivated to use GRAIL as the core positioning technology in our research and deployment because the current implementation of the system supports vari- 
ous wireless RFID tags and can be extended to include other tracking information in addition to the RSS such as mobility and proximity. GRAIL already supports WiFi tags, special active pipsqueak tags [4], ZigBee tags and Bluetooth phones. It achieves an average tracking accuracy of roughly room-level $10-15 \mathrm{ft}$. This performance is also consistent with most commercial and academic indoor RTLS systems, as shown in earlier studies that cross-evaluated different positioning solvers in different environments, e.g., [8], among others.

The basic system components of GRAIL are as follows. Landmarks observe packet traffic and send the packets headers along with information about the RSS to a centralized location, called the server. In order to localize a set of devices, the sever processes all the received packets, and forwards summarized observations to a solver program. The solver is what actually estimates the positions of the device radios. Finally, the results is displayed on a Graphical User Interface (GUI) to the user or used for higher level applications through an Application Programming Interface (API). GRAIL is thus built around four components: a server, a solver, a GUI/API, and landmarks. For more information about GRAIL the reader is referred to [5], [3], [9].

\section{Clinical Tracking Pilot Study}

In this section we describe early preliminary two-day experiments and findings at the outpatient Cancer clinic of the Penn State Hershey Medical Cancer (PSHMC) and its impact on decision making at the administrative level. The goal was to test the expected tracking accuracy in such an unpredictable environment and whether our RTLS technology is able to measure durations of different atomic clinical activities that involve one mobile object (a nurse, a physician, a patient or a piece of equipment). Next, we experimented with the utility of this data in inferring more complex activities and events, which we show in the next section.

The clinic is approximately $80 \times 96 \mathrm{ft}$. The layout of the clinic is shown in Figure 1. It includes a reception area, multiple treatment rooms, a nurses room as well as a physicians' consultation room. We deployed 7 GRAIL landmarks which are shown as red circles. Those are single board computers populated with a radio capturing code. Typical functions performed in the clinic include tests, consultations, and treatments, and there are no overnight patients. In day 1 we installed the GRAIL system software, deployed the landmarks, and created radio signal maps for the floor. The tracking experiments were conducted over the next day.

\section{A. The Need}

Based on our interviews, the clinic staff are generally interested in improving the efficiency of the clinic and the waiting time of their patients. Initially they wanted: (a) to figure out whether they should invest in purchasing or renting extra equipment, Specifically "Dinamap" portable vital signs monitors. The clinic has 5, and each one costs about $\$ 5,400$ including the pole. They maintain one per hallway. (b) For them, another factor that introduces significant delays is wasted time locating misplaced paper medical charts (and other resources). This is a critical problem since they are not expecting to upgrade their systems to EMR within the next two or three years. According to staff members, charts are supposed to move through the center with the patients, because a patient cannot be treated unless the chart is available. Occasionally, however, charts are misplaced and the nurses spend time looking for them. While the chart is unavailable the patient has to wait. These delays can become especially acute when the chart is in another unit of the hospital. (c) The third issue is the need to quantify overhead and unscheduled activities as they occur. Once quantified, the next step would be to cut on those activities using different strategies such as relocating or even hiring more needed staff.

\section{B. Experiments}

In order to address the staff concerns we performed 3 experiments: (1) tracking Dinamap monitors, (2) tracking paper charts and (3) simulating a physician's movement. We used pipsqueak wireless RFID tags [4] for the first two experiments and a small PDA/PC for the latter (WiFi tags achieve the same results).

Equipment Tracking: We attached an RFID tag to the bottom of each Dinamap monitor. We tracked each one for a few hours and recorded the ground truth manually every 5 to 10 minutes. Dinamaps residing in hallways at a specific time were labeled as "nonutilized" while those inside treatment rooms were considered "utilized" or "in use". We experimented with tags at two different output power to measure the accuracy of tracking in both cases. Figure 1 (a), (b) shows the tracking results on a map of the clinic for two dinamaps. The blue ellipses represent the location estimate. The size of the ellipse is scaled to the confidence of the result; the center of each ellipse shows the most likely position, and the size is 1 standard deviation in the $\mathrm{X}$ and $\mathrm{Y}$ dimensions. The red " $\mathrm{X}$ " is the true location, or ground truth. A red line connects the center of the estimated ellipse with the ground truth.

Paper Chart Tracking: We tracked medical charts moving around the clinic in real time. We did not use actual charts for privacy reasons, but instead moved a folder full of 200 sheets of paper, identical to a real chart, along a path similar to how a chart would move. The ground truths were collected manually every time we moved the chart. Figure 1 (c) shows the results for 


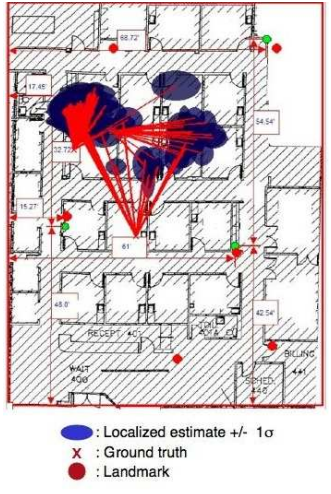

(a)

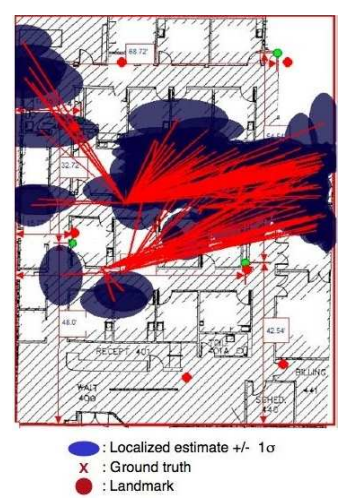

(b)

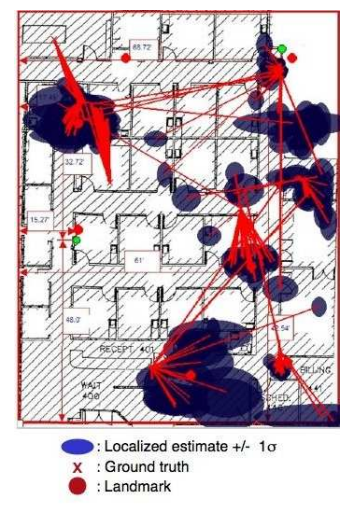

(c)

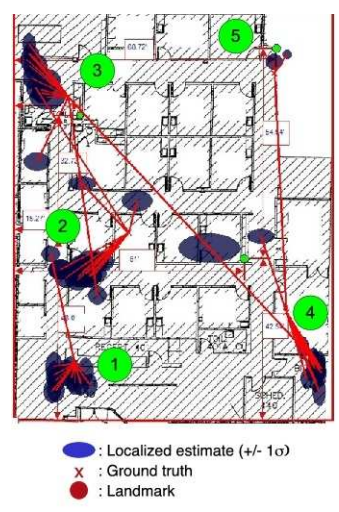

(d)

Figure 1. Performance for 10db pipsqueak (a), 1db pipsqueak (b), patient chart (c), and physician PDA (d).

this experiment. The average accuracy was 10-15 feet, although there were significant outliers due to lags in the RTLS system; when a chart moves from one location to another it can take up to 1 minute before the localization system "catches up" to an area close to the chart's true position. This was specifically due to the low tracking frequency we used, once every 30 seconds, which we fixed for the activity experiments we describe next.

Physician Tracking: We tracked a physician's movement inside the clinic. The goal was to test whether tracking alone can predict atomic clinical events which involve one mobile object from locations. We used a PDA-like device, a very small WiFi sub-notebook computer (a 7 inch eeePC). A typical movement scenario is to (1) Pick up patient at reception, (2) Consult a nurse in the nurses' room, (3) Examine patient in examination room, (4) Visit the physicians' consulting room, and (5) Drop off the patient's chart at another side desk. Figure 1 (d) shows the results. The figure is annotated with the ground truth locations of the activities.

Findings and Discussion: We found that the clinic inventory of 5 Dinamap devices is too high and often those devices are under-utilized. Indeed, 2 or 3 Dinamaps are sufficient for the workload on the days we performed our pilot, thus a reduction of $40-60 \%$ can be performed without affecting the clinic throughput. Moreover, we were able to track the medical charts with adequate accuracy most of the time. We were also able to identify the simple clinical events involving physicians and their duration as in (1), (2), (3) and (5) from Figure 1 (d). The accuracy was not good enough to predict whether the physician was in the consultation room (the one marked as 4) or in the billing room right next to it.

Localization results for $\mathrm{WiFi} / \mathrm{PDA}$ had 10ft error on average while the average error for the pipsqueak tags was $10-15 \mathrm{ft}$, which is a room-level accuracy. In some cases, as in tracking Dinamap devices the performance was much worse. We used pipsqueak tags programmed at different power levels (1db and 10db). The higher the $\mathrm{db}$ the more quickly the tag battery drains. We found tags with $1 \mathrm{db}$ power level too weak and yield poor performance as shown in Figure 1 (b). The weak signal problem is made worse because we needed to hide the tags under the Dinamap monitors, which further attenuated the signal. A potential solution is to attach the tags to the poles, and have external antennas.

\section{EVEnt Detection Experiments}

This section describes a second set of experiments which emulated an Emergency Department (ED). The goal is to detect clinical activities from the tracking traces given a simple model of them. We start with an overview of the ED workflow at the RWJUH hospital in New Jersey. The model was abstracted after visits to several emergency departments as well as interviews with nurses and physicians at different hospitals. We then describe how we automatically deduced the activities in the workflow.

Figure 2 is a more formal workflow graph representation of a patient's visit to the ED, while Figure 3 shows the emulated ED map. The goal of our approach is to automatically detect when the various workflow events, represented by nodes in the graph, have been accomplished from the spatial temporal movements occurring on the map shown by Figure 3.

ED Workflow Abstraction: Figure 2 shows the first step when a patient arrives is that he is seen by a greeter, who takes the patient's name. The patient then waits to be interviewed by a triage nurse in a triage room. After triage, the patient further waits for a bed assignment. These are nodes 1-2 in the graph.

At the bedside, 3 care providers visit the patient: A registrar, a physician, and a primary nurse. These can occur in any order, and are shown as nodes 3-5. After having been seen by all 3 , a workup event occurs, which is the logical join of the three proceeding steps. The next set of tasks, lab, consult, and radiology, depend on the patient's condition as determined by the physician's 


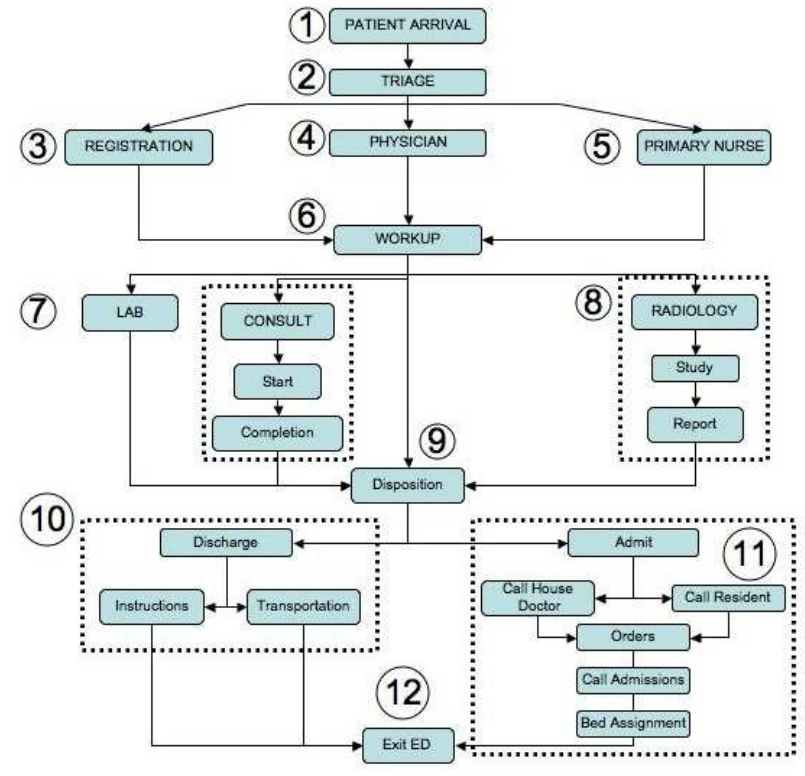

Figure 2. Patient Flow Diagram. Each box is a task and edges represent dependencies. RTLS can identity 12 subprocess labeled with circles.

assessment in task 4. The Lab tasks are typically blood and urine tests. The radiology task is typically an Xray. The consult task is when the ED physician requires outside expertise, for example, a psychiatrist.

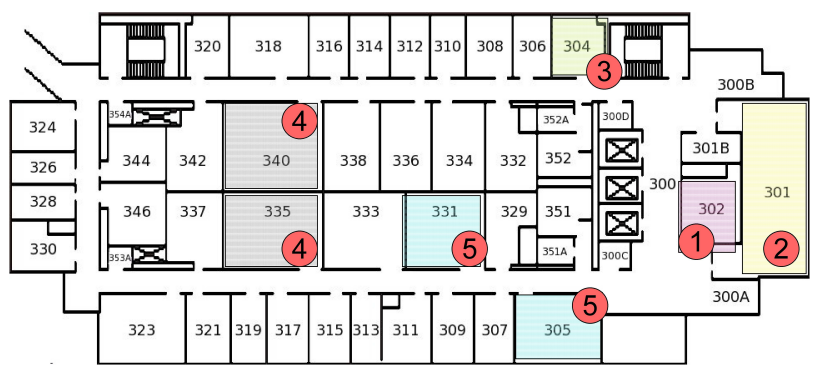

Figure 3. The ED experiment floor annotated with the activities locations. (1) Reception, (2) Waiting, (3) Triage, (4) Exam rooms, (5) Physicians/Nurses/Consultation.

Disposition, task 9, is when all the above tasks are completed and the ED physician makes a decision if the patient is well enough to be discharged, or must be admitted to the hospital. Disposition is also a join of the other tasks, although these are optional. For example, a patient requiring a few stitches would not require any of the lab, consult or radiology steps. When a patient is discharged, non-ambulatory patients require transport. Ambulatory patients can simply leave the ED with instructions for follow up care, if necessary. Admitted patients go through one of two possible paths: either a resident is contacted for admission orders or their own physician, i.e., the house doctor. In either case, admission orders are required, which results in a hospital bed assignment. The patient hence remains boarded in the ED until the hospital makes the bed assignment.

Scripted Events: We ran a set of human scripts/scenarios to test the RTLS utility for ED activity detection. Although hospitals are the most realistic environment for the study we ran the experiments in an emulated environment at the CoRE building at Rutgers University. Specifically, due to difficulty of replicating the experiment multiple times, the effort, time and expense of a preliminary deployment in a real hospital, and the potential impact on actual hospital operation. The CoRE building is a good approximation of the physical space in a hospital. It shares with hospitals a similar modern steel-frame construction, has many smaller spaces partitioned with walls, and a high density of equipment, see Figure 3.

We increased the frequency of tracking (i.e., number of location predictions within a time window) and tuned the RTLS system so that we do not run into lags as in the first study. We also used better RFID tags with improved exposed antennas and transmission power, which yielded better wireless radio signals. We used a denser deployment of the landmark hardware to minimize areas where there are no wireless signal readings.

The scripts were used to direct human motions in a manner representative of an actual ED, i.e., actors moved around in a manner similar to real ED scenarios. We modeled several scenarios, including (1) base treatment case, (2) burst at triage, (3) leave without being seen, (4) lost lab work, and (5) misplaced patient. The human flows generated wireless RFID traces which we used to evaluate the current tracking system with respect to identifying a predefined set of clinical events.

We ran different experiments to test different scenarios. Here we describe only one due to the limited space. Figure 4 shows snapshots of the experiment. Again, the size of the ellipse is scaled to the confidence of the result while its center shows the most likely position. The floor is approximately $85 \times 200 \mathrm{ft}$. Each actor's “location prediction" has a different color; the red is for triage nurse located in room 304 while the orange is for the receptionist located in room 302. The remaining colors are for patients that started accumulating in the waiting area (room 301) one after the other and after they check in with the receptionist. Notice the overlap between the orange ellipse and the patients' ellipses while talking with the receptionist in snapshots 1,2 , as well as the overlapped ellipses in room 301 when one of the patients is triaged in snapshot 3. This patient then walks (not shown) in the corridor to a checkup room (room 335), where s/he finally waits for treatment, snapshot 4 . Notice the blue colored patient disappeared in snapshot 4, as he left the floor without being treated.

Event Prediction: We initially used simple statistical models to predict events. They are probabilistic because 


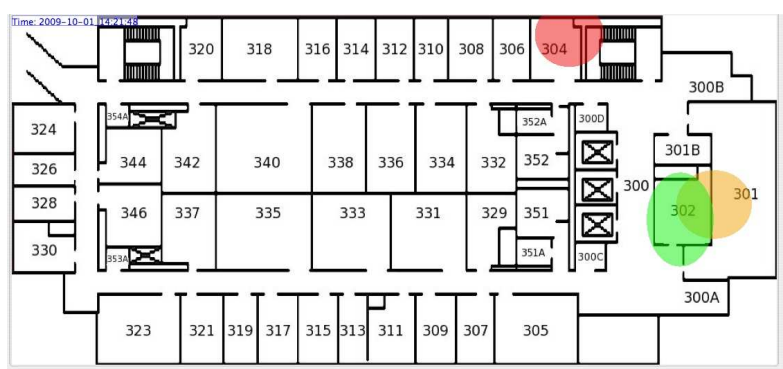

snapshot (1) Patient 1 checking in with receptionist

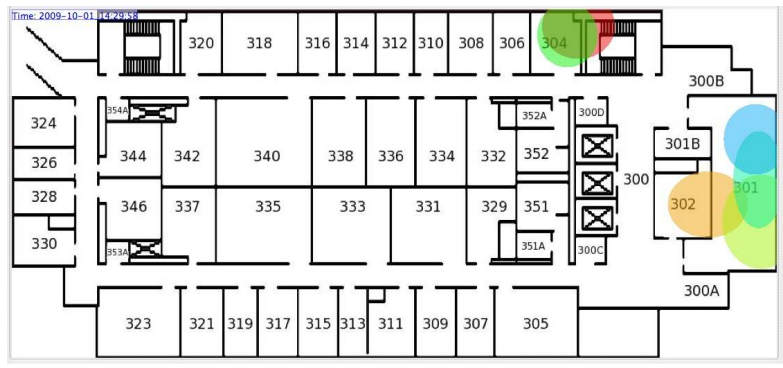

snapshot (3) Patient 1 triaged and 2 more patients waiting

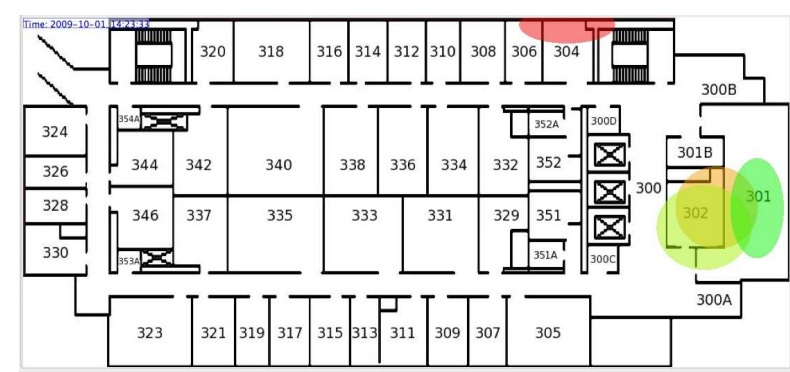

snapshot (2) Patient 2 checking in with receptionist while 1 waited

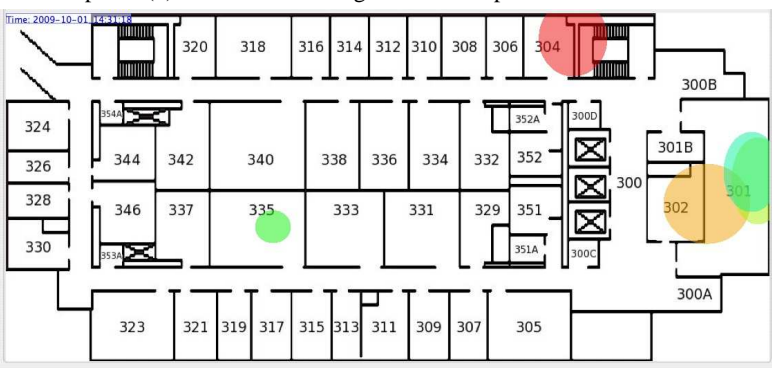

snapshot (4) Patient 1 in checkup room and patient 4 left without treatment

Figure 4. Patients Buildup Experiment. Triage nurse in Red, receptionist in Orange, patients in shades of Green and Blue.

they are extracted from RTLS locations, which are probabilistic due to uncertainties and imperfections in their observed RSS evidences. Specifically, the location $(x, y)$ of a patient on the ED floor is approximated by a joint probability distribution of $(x, y)$ at each point or tile on the floor. The GRAIL RTLS assumes Gaussian distributions and outputs the most probable/likely position along with a surrounding confidence area extended from the position prediction variance, as noted in the ellipse description above. Our approach to deducing event occurrences is spatio-temporal. It involves co-location of mobile objects (patients, nurses and physicians) and/or pieces of equipment in specific areas over an extended period of time. To detect a "patient waiting" event, e.g., we compute the probability that the bulk of the location distribution lies inside the waiting area $n$ out of the $m$ tracking predictions within a period of $t$ minutes. The parameters $n, m, t$ are estimated from the ED operation.

$$
p\left(\text { waiting, } t=t_{i}\right)=\sum_{\text {waiting area tiles }} p(x, y)
$$

We assume the location predictions over time are independent for simplicity. We accept or reject an occurrence based on threshold probability parameters approximated from the RTLS performance.

$$
p(\text { waiting event })=\frac{\sum_{t_{i}=t_{0}}^{t_{m}} p\left(\text { waiting, } t=t_{i}\right)}{m}
$$

Other more complicated events such as "triage" involves both the co-location probability as well as the occurrence of other prior events, specifically waiting and/or being greeted. In order to combine these probabilities together we utilize Bayes rule, in Equation 3, to compute the posterior distribution of triage given the probability distribution of the waiting event happening prior to it, $p\left(\right.$ triage at $t_{i} \mid$ waiting at $\left.t<t_{i}\right)$.

$$
p(\theta \mid x)=\frac{\text { likelihood } \times \text { prior }}{\text { evidence }}=\frac{p(x \mid \theta) p(\theta)}{p(x)}
$$

Which can be rewritten as follows. Notice the individual probabilities are computed from the co-location information as in Equations 1, 2 and from observing the probability of triage occurring right after waiting from collected training information.

$\frac{p\left(\text { waiting at } t<t_{i} \mid \text { triage at } t_{i}\right) \times p\left(\text { waiting at } t<t_{i}\right)}{p\left(\text { triage at } t_{i}\right)}$

Figure 4 however shows the drawback of using the location information alone to deduce some of the events. Specifically, because the receptionist desk resides right next to the waiting area it is sometimes hard to distinguish between a patient interacting with her/him or just waiting. This is an inherent limitation of any RTLS system that uses wireless RFIDs. One of our future work goals is hence to introduce other sources of information such as mobility and proximity into the model and use a statistical Bayesian networks to resolve this confusion. We will generalize the above Bayes rules to a Bayesian Graphical Model that combines information from multiple evidences (mobility and proximity) in addition to the spatio-temporal location information.

Dynamic Workflow Management: Before concluding we emphasize that the goal of our research is to study the utility of RTLS tracking in improving the efficiency of operation. The last piece of the overall picture is thus, given we can deduce the clinical activities, how 
do we model the workflow and detect, say a surge/burst of patients in real-time? Furthermore, how to alter the workflow, and, e.g., make staffing level decisions, to improve it. This is an area that would offer a significant advance in this field. We ran preliminary simulations from our trace-driven clinical tracking experiments using the Arena simulation tool (www.arenasimulation.com). We approximated models of the clinical activities from their spatio-temporal approach occurrences. We modeled patients and care providers as entities and approximated each activity duration/service time. Inaccuracies and irregularities in predictions were accounted for by adding variances. Our future work will focus on extending and validating these preliminary models from long term clinical data that we will collect at a local hospital.

\section{Ongoing And Future Work Agenda}

Our future research plan can be broadly summarized as follows. We will conduct thorough long term experiments in a local hospital in which we will attach RFID tags to patients, care givers, and equipment. We will research models to translate mobility, and location data into pre-defined ED activities, including waiting, preparation, treatment, and discharge. We will also experiment with more complex approaches that involve proximity from other tags worn by other care givers. Using this data we plan to perform an in depth analysis of the workflow and resource re-allocation scenarios. Another direction is to build a software layer on top of the GRAIL RTLS to display alerts when the real time schedule inside the clinic starts diverting dramatically from its historical model. This layer may enable different actions such as sending text message notifications to patients about delays in their scheduled appointments.

\section{RELATED WORK}

There are two classes of related work we cover here. First, there are numerous, mostly commercial RTLS systems that are geared for health-care inventory control applications [1], [7], [2]; a more general survey of such applications can be found in [10]. Our initial results show that the position accuracy and latency needed for workflow detection are significantly greater than that for inventory management application, which operate on longer-timescales.

A second body of related work focuses on health care workflow. These systems to date have not been aggressive in the application of RTLS technologies. The closest work to ours in that area is [11], which proposed integrating numerous hospital systems into a workflow engine. However, the event detection driven by the tracking subsystem was more limited in scope than our approach, such as checking into a unit of the hospital. Because the assumed technology was passive
RFID tags, and these required patients and providers pass close to portals, the events were thus more limited in scope than our approach where we assume better spatial-temporal resolution. It is an interesting open question, however, as to the value of the additional spatial-temporal coverage gains over the close reader proximity approaches provided with passive RFID as well as the cost and scalability factors while deploying the RTLS system.

\section{CONCLUSION}

In this work we demonstrated the utility of RTLS in modeling and managing workflow in hospitals using the GRAIL RTLS system. Specifically, we described our preliminary experiments to convert tracking data into workflow processes. We also discussed various lessons we learned from our pilots.

\section{ACKNOWLEDGMENT}

This work was supported in part by NSF SBIR (no. 0740629), Rutgers Seed Fund grant for collaborative Computing Research, and RWJ Foundation.

\section{REFERENCES}

[1] Aeroscout system. White papers, www.aeroscout.com.

[2] Awarepoint. White papers, www.awarepoint.com.

[3] Grail documentation and source code: project grail-rtls. http://grailrtls. sourceforge. net.

[4] Inpoint systems. white paper, http://inpointsys.com.

[5] Y. Chen, G. Chandrasekaran, E. Elnahrawy, J.-A. Francisco, K. Kleisouris, X. Li, R. P. Martin, R. S. Moore, and B. Turgut. GRAIL: A genenral purpose localization system. In Sensor Review, 2008.

[6] Y. Chen, J.-A. Francisco, K. Kleisouris, H. Xue, R. P. Martin, E. Elnahrawy, and X. Li. GRAIL: general realtime adaptable indoor localization. In ACM SenSys, 2006.

[7] Ekahau, Inc. The Ekahau Positioning Engine. White papers, www.ekahau.com, July 2003.

[8] E. Elnahrawy, X. Li, and R. P. Martin. The limits of Localization Using Signal Strength: A Comparative Study. In IEEE SECON, 2004.

[9] E. Elnahrawy and R. P. Martin. On class of localization algorithms using received signal strength. In Localization Algorithms and Strategies for Wireless Sensor networks by Guoqiang Mao and Baris Fidan. IGI Global, 2009.

[10] P. Fuhrer and D. Guinard. Building a smart hospital using RFID technologies. In European Conference on eHealth $(E C E H)$, Fribourg, Switzerland, Oct. 2006.

[11] J.-C. Kuhr, J. Pretzel, D. A. Vagts, and L. Aldred. Integrating humans, devices, and events in clinical workflow processes. In Business Process Management Workshops, 2008. 